\title{
HEARING THE SHAPE OF A COMPACT RIEMANNIAN MANIFOLD WITH A FINITE NUMBER OF PIECEWISE IMPEDANCE BOUNDARY CONDITIONS
}

\author{
E.M.E. ZAYED
}

Mathematics Department

Faculty of Science

Zagazig University

Zagazig, EGYPT

(Received June 8, 1994 and in revised form March 21,1996)

ABSTRACT. The spectral function $\Theta(t)=\sum_{i=1}^{\infty} \exp \left(-t \lambda_{\jmath}\right)$, where $\left\{\lambda_{\jmath}\right\}_{j=1}^{\infty}$ are the eigenvalues of the negative Laplace-Beltrami operator $-\Delta$, is studied for a compact Riemannian manifold $\Omega$ of dimension " $k$ " with a smooth boundary $\partial \Omega$, where a finite number of piecewise impedance boundary conditions $\left(\frac{\partial}{\partial n_{1}}+\gamma_{2}\right) u=0$ on the parts $\partial \Omega_{\imath}(i=1, \ldots, m)$ of the boundary $\partial \Omega$ can be considered, such that $\partial \Omega=\bigcup_{\imath=1}^{m} \partial \Omega_{\imath}$, and $\gamma_{\imath}(i=1, \ldots, m)$ are assumed to be smooth functions which are not strictly positive

KEY WORDS AND PHRASES: Inverse problem, Laplace Beltrami operator, eigenvalues, spectral function, Riemannin manifold

1991 AMS SUBJECT CLASSIFICATION CODES: 35K, 35P, 58G32

\section{INTRODUCTION}

The underlying problem is to determine the geometry of a compact $k$-dimensional smooth Riemannian manifold $\Omega$ with metric tensor $g=\left(g_{\alpha \beta}\right)$, from a complete knowledge of the eigenvalues for the negative Laplace-Beltrami operator $-\triangle=-\frac{1}{\sqrt{\operatorname{det} g}} \frac{\partial}{\partial x_{\alpha}}\left[g^{\alpha \beta} \sqrt{\operatorname{det} g} \frac{\partial}{\partial x_{\beta}}\right]$ where $g^{-1}=\left(g^{\alpha \beta}\right)$

Let $\Omega$ be a compact Riemannian manifold of dimension " $k$ " with a smooth boundary $\partial \Omega$ Suppose that the eigenvalues

$$
0<\lambda_{1} \leq \lambda_{2} \leq \lambda_{3} \leq \ldots \leq \lambda_{\jmath} \leq \ldots \rightarrow \infty \text { as } j \rightarrow \infty,
$$

are known exactly for the eigenvalue equation

$$
(\Delta+\lambda) u=0 \text { in } \Omega
$$

together with the impedance boundary condition

$$
\left(\frac{\partial}{\partial n}+\gamma\right) u=0 \text { on } \partial \Omega
$$

where $\frac{\partial}{\partial n}$ denotes differentiation along the inward pointing normal to $\partial \Omega$ and $\gamma$ is a smooth function which is not strictly positive

Hsu [1] has investigated problem (1.2)-(1 3) and has determined the geometric quantities associated with the manifold $\Omega$ from the asymptotic expansion of the spectral function 


$$
\Theta(t)=\sum_{\jmath=1}^{\infty} \exp \left(-t \lambda_{\jmath}\right) \quad \text { as } \quad t \rightarrow 0
$$

Problem (1.2)-(1 3) has been investigated by many authors (see, for example, Mckean and Singer [3] and Hsu [1]) if $\gamma \equiv 0$ (Neumann problem) and have shown that

$$
(4 \pi t)^{k / 2} \Theta(t)=a_{0}+a_{1} t^{1 / 2}+a_{2} t+a_{3} t^{3 / 2}+0\left(t^{2}\right) \text { as } t \rightarrow 0
$$

where

$$
\begin{aligned}
& a_{0}=|\Omega|, \\
& a_{1}=\frac{\sqrt{\pi}}{2}|\partial \Omega|, \\
& a_{2}=\frac{1}{6} \int_{\Omega} K(x) d x-\frac{1}{3} \int_{\partial \Omega} \operatorname{tr} H(z) d z,
\end{aligned}
$$

and

$$
a_{3}=\sqrt{\pi} \int_{\partial \Omega}\left\{\frac{1}{12} K^{\partial \Omega}(z)-\frac{37}{192}[\operatorname{tr} H(z)]^{2}+\frac{29}{96} \operatorname{tr} H^{2}(z)+\frac{1}{8} \operatorname{Ric}(n)(z)\right\} d z .
$$

In these formulae, $|\Omega|$ is the (Riemannian) volume of $\Omega,|\partial \Omega|$ is the (Riemannian) surface area of $\partial \Omega, K(x)$ is the scalar curvature of $\Omega$ at $x, H(z)$ is the second fundamental form of the boundary $\partial \Omega$, $K^{\partial \Omega}(z)$ is the scalar curvature of $\partial \Omega$ (equipped with the induced metric) at $z, \operatorname{Ric}(n) z$ is the Ricci curvature of $\Omega$ at $z$ in the normal direction " $n$ " of the boundary $\partial \Omega$, and $\operatorname{tr} H(z)$ is the mean curvature of $\partial \Omega$

The object of this paper is to discuss the following more general inverse problem Suppose that the eigenvalues (1 1 ) are known exactly for the eigenvalue equation (1.2) together with the following piecewise smooth impedance boundary conditions

$$
\left(\frac{\partial}{\partial n_{\imath}}+\gamma_{\imath}\right) u=0 \text { on } \partial \Omega_{\imath}(i=1, \ldots, m),
$$

where the boundary $\partial \Omega$ of $\Omega$ consists of a finite number of the parts $\partial \Omega_{\imath}(i=1, \ldots, m)$ such that $\partial \Omega=\bigcup_{\imath=1}^{m} \partial \Omega_{\imath}$, while $\frac{\partial}{\partial n_{i}}$ denote differentiations along the inward pointing normals to $\partial \Omega_{\imath}$ and $\gamma_{\imath}$ are assumed to be smooth functions defined on $\partial \Omega_{\imath}$ which are not strictly positive.

The basic problem is that of determining the geometry of the manifold $\Omega$ as well as the impedance functions $\gamma_{2}(i=1, \ldots, m)$ from the asymptotic expansion of the spectral function

$$
\Theta(t)=\sum_{\jmath=1}^{\infty} \exp \left(-t \lambda_{\jmath}\right) \quad \text { as } \quad t \rightarrow 0 .
$$

Note that the main problem (1.2) and (1.6) has been discussed recently by Zayed and Younis [4] and Zayed [5-7] in the case where $\Omega$ is a general simply connected bounded domain in $R^{k}(k=2$ or 3$)$ with a smooth boundary $\partial \Omega$ and $\gamma_{2}(=1, \ldots, m)$ are positive constants

\section{STATEMENT OF RESULTS}

THEOREM. Let $\left|\partial \Omega_{\imath}\right|(i=1, \ldots, m)$ be the (Riemannian) surface areas of the parts $\partial \Omega_{\imath}(i=1, \ldots, m)$ of the boundary $\partial \Omega$ respectively. Let $K^{\partial \Omega_{1}}(z),(i=1, \ldots, m)$ be the scalar curvatures of the parts $\partial \Omega_{\imath}(i=1, \ldots, m)$ of $\partial \Omega$ respectively. Let $\operatorname{Ric}\left(n_{\imath}\right)(z)$ be the Ricci curvatures of $\partial \Omega_{\imath}$ at $z$ in the normal directions $n_{\imath}$ of the parts $\partial \Omega_{\imath}(i=1, \ldots, m)$ of $\partial \Omega$. Then the results of problem $(12)$ and (16) can be written in the form 


$$
(4 \pi t)^{k / 2} \Theta(t)=a_{0}+a_{1} t^{1 / 2}+a_{2} t+a_{3} t^{3 / 2}+0\left(t^{2}\right) \text { as } t \rightarrow 0
$$

where

$$
\begin{aligned}
& a_{0}=|\Omega| \\
& a_{1}=\frac{1}{2} \sqrt{\pi} \sum_{i=1}^{m}\left|\partial \Omega_{2}\right|, \\
& a_{2}=\frac{1}{6} \int_{\Omega} K(x) d x-\frac{1}{3} \sum_{i=1}^{m} \int_{\partial \Omega_{i}}\left[\operatorname{tr} H(z)+6 \gamma_{i}(z)\right] d z,
\end{aligned}
$$

and

$$
\begin{aligned}
a_{3}=\sqrt{\pi} \sum_{z=1}^{m} \int_{\partial \Omega_{1}}\left\{\frac{1}{12}\right. & K^{\partial \Omega_{\imath}}(z)-\frac{37}{192}[\operatorname{tr} H(z)]^{2}+\frac{29}{96} \operatorname{tr} H^{2}(z) \\
& \left.+\frac{1}{8} \operatorname{Ric}\left(n_{\imath}\right)(z)+\frac{1}{2} \gamma_{\imath}(z) \operatorname{tr} H(z)+\gamma_{\imath}^{2}(z)\right\} d z .
\end{aligned}
$$

Note that the results of Neumann conditions on $\partial \Omega_{\imath}$ are obtained from (2 1$)$ by setting $\gamma_{\imath} \equiv 0$ $(i=1, \ldots, m)$ which are in agreement with the results $(15)$ of Neumann conditions on $\partial \Omega$

REMARK 1. If $\Omega$ is a bounded domain in $R^{2}$ with a smooth boundary $\partial \Omega$, then $K^{\partial \Omega_{1}}(z)=0$, $\operatorname{Ric}\left(n_{\mathrm{z}}\right)(z)=0, \operatorname{tr} H^{2}(z)=c^{2}(z), \operatorname{tr} H(z)=-c(z)$ where $c(z)$ is the curvature of $\partial \Omega$ at $z$ and if $\gamma_{z}$ are positive constants then, we get the result of Zayed [5] when $i=1,2$ and the result of Zayed and Younis [4] when $i=1, \ldots, m$.

REMARK 2. If $\Omega$ is a bounded domain in $R^{3}$ with a smooth surface $\partial \Omega$, then $K^{\partial \Omega_{1}}(z)=2 k_{1} k_{2}$, $\operatorname{Ric}\left(n_{2}\right)(z)=0, \operatorname{tr} H^{2}(z)=k_{1}^{2}+k_{2}^{2}, \operatorname{tr} H(z)=-k_{1}-k_{2}$, where $k_{1}$ and $k_{2}$ are the two principal curvatures of the boundary surface $\partial \Omega$ at $z$ and if $\gamma_{2}$ are positive constants, then we get the result of Zayed [6] when $i=1,2$ and also the result of Zayed [7] when $i=1, . ., m$

\section{CONSTRUCTION OF RESULTS}

Following the method of Kac [2] and Hsu [1], it is easily seen that $\Theta(t)$ associated with problem (1.2) and (1 6) is given by

$$
\Theta(t)=\int_{\Omega} G(t, x, x) d x,
$$

where the heat kernel $G(t, x, y)$ is defined on $(0, \infty) \times \bar{\Omega} \times \bar{\Omega}$, which satisfies the following

For fixed $x \in \bar{\Omega}$, it satisfies in $t, y$ the heat equation

$$
\left(\frac{\partial}{\partial t}-\triangle_{y}\right) G(t, x, y)=0
$$

and the piecewise impedance boundary conditions

$$
\left[\frac{\partial}{\partial n_{\imath y}}+\gamma_{\imath}(y)\right] G(t, x, y)=0 \quad \text { on } \quad \partial \Omega_{\imath}(i=1, \ldots, m),
$$

and the initial condition

$$
\lim _{t \rightarrow 0} G(t, x, y)=\delta(x-y)
$$

where $\delta(x-y)$ is the Dirac delta function located at the source point $x=y$ Note that in (3 2)-(3 3) the subscript " $y$ " means that the derivatives are taken in $y$-variables.

Thus by the superposition principle of the heat equation, we write

$$
G(t, x, y)=G_{N}(t, x, y)+\chi(t, x, y)
$$


where $G_{N}(t, x, y)$ is the Neumann heat kernel on $\Omega$ which satisfies the heat equation

$$
\left(\frac{\partial}{\partial t}-\triangle_{y}\right) G_{N}(t, x, y)=0
$$

and the piecewise Neumann boundary conditions

$$
\frac{\partial}{\partial n_{\imath y}} G_{N}(t, x, y)=0 \text { on } \partial \Omega_{\imath}(i=1, \ldots, m),
$$

and the initial condition

$$
\lim _{t \rightarrow 0} G_{N}(t, x, y)=\delta(x-y)
$$

while $\chi(t, x, y)$ satisfies the heat equation

$$
\left(\frac{\partial}{\partial t}-\triangle_{y}\right) \chi(t, x, y)=0
$$

and the piecewise boundary conditions

$$
\frac{\partial}{\partial n_{z y}} \chi(t, x, y)=-\gamma_{z}(y) G(t, x, y) \quad \text { on } \quad \partial \Omega_{\imath}(i=1, \ldots, m)
$$

and the initial condition

$$
\lim _{t \rightarrow 0} \chi(t, x, y)=0 .
$$

Now, the solution of problem (3.9), (3.10) and (3 11) is given by

$$
\chi(t, x, y)=-\sum_{\imath=1}^{m} \int_{0}^{t} d s \int_{\partial \Omega_{1}} G_{N}(t-s, x, z) \gamma_{\imath}(z) G(s, z, y) d z
$$

From (3.5) and (3.12) we have the integral equation

$$
G(t, x, y)=G_{N}(t, x, y)-\sum_{i=1}^{m} \int_{0}^{t} d s \int_{\partial \Omega_{1}} G_{N}(t-s, x, z) \gamma_{\imath}(z) G(s, z, y) d z .
$$

On applying the iteration method (see [5]) to the integral equation (3 13) we obtain the series

$$
G(t, x, y)=\sum_{r=0}^{\infty}(-1)^{r} F_{r}(t, x, y)
$$

where

$$
F_{0}(t, x, y)=G_{N}(t, x, y)
$$

and

$$
F_{r}(t, x, y)=\sum_{\imath=1}^{m} \int_{0}^{t} d s \int_{\partial \Omega_{1}} G_{N}(t-s, x, z) \gamma_{\imath}(z) F_{r-1}(s, z, y) d z, \quad(r=1,2, \ldots) .
$$

From (3.1), (3 5), (3.14) and with the help of the following well known estimate (see [1], [3])

$$
(4 \pi t)^{k / 2} \sum_{r=3}^{\infty} \int_{\Omega}\left|F_{r}(t, x, x)\right| d x=0\left(t^{2}\right) \quad \text { as } t \rightarrow 0,
$$

we deduce as $t \rightarrow 0$ that

$$
\Theta(t)=\Theta_{N}(t)-\int_{\Omega} F_{1}(t, x, x) d x+\int_{\Omega} F_{2}(t, x, x) d x+0\left(t^{(4-k) / 2}\right),
$$


where $\Theta_{N}(t)=\int_{\Omega} G_{N}(t, x, x) d x$, which has the same asymptotic expansion (2 1) with $\gamma_{2} \equiv 0$

The problem now is to study the integrals of $F_{r}(t, x, x),(r=1,2)$ over the manifold $\Omega$

LEMMA 1. We have as $t \rightarrow 0$,

$$
\begin{aligned}
(4 \pi t)^{k / 2} \int_{\Omega} F_{1}(t, x, x) d x= & 2 t \sum_{i=1}^{m} \int_{\partial \Omega_{i}} \gamma_{z}(z) d z \\
& -\frac{1}{2} \sqrt{\pi} t^{3 / 2} \sum_{i=1}^{m} \int_{\partial \Omega_{\imath}} \gamma_{\imath}(z) \operatorname{tr} H(z) d z+0\left(t^{2}\right) .
\end{aligned}
$$

PROOF. The definition of $F_{1}(t, x, x)$ and the Chapman-Kolmogorov equation of the heat kernel imply

$$
\int_{\Omega} F_{1}(t, x, x) d x=t \sum_{\imath=1}^{m} \int_{\partial \Omega_{\imath}} G_{N}(t, z, z) \gamma_{\imath}(z) d z .
$$

Let us now introduce the following well known estimate of the Neumann heat kernel (see [1])

$$
(4 \pi t)^{k / 2} G_{N}(t, z, z)=2\left[1-\frac{1}{4} \sqrt{\pi t} \operatorname{tr} H(z)\right]+0(t) \text { as } t \rightarrow 0
$$

which is valid uniformly in $z \in \partial \Omega_{\imath}(i=1, \ldots, m)$.

On inserting (3.20) into (3.19) we arrive at the proof of Lemma 1

LEMMA 2. We have as $t \rightarrow 0$

$$
(4 \pi t)^{k / 2} \int_{\Omega} F_{2}(t, x, x) d x=\sqrt{\pi} t^{3 / 2} \sum_{i=1}^{m} \int_{\partial \Omega_{i}} \gamma_{\imath}^{2}(z) d z+0\left(t^{2}\right) .
$$

PROOF. From the definition of $F_{2}(t, x, x)$ and with the help of the expression of $F_{1}(t, x, x)$ we deduce that

$$
\int_{\Omega} F_{2}(t, x, x) d x=\sum_{z=1}^{m} \int_{0}^{t}(t-u) d u \int_{\partial \Omega_{2}} \gamma_{\imath}(z) d z \int_{\partial \Omega_{\imath}} G_{N}(t-u, z, y) \gamma_{\imath}(y) G_{N}(u, y, z) d y .
$$

We replace $\gamma_{2}(y)$ in the above integral by $\gamma_{2}(z)+0(|z-y|)$ and split the integral into two integrals accordingly Using the following estimate for the Neumann heat kernel There exist positive constants $t_{0}, c_{1}$ such that for all $t<t_{0},(x, y) \in \bar{\Omega} \times \bar{\Omega}$,

$$
G_{N}(t, x, y) \leq c_{1} t^{-k / 2} \exp \left\{-\frac{|x-y|^{2}}{c_{1} t}\right\}
$$

we deduce that

$$
\int_{\partial \Omega_{1}}|z-y| G_{N}(t-u, y, z) G_{N}(u, z, y) d y \leq c_{1}[u(t-u)]^{-k / 2} \int_{R^{k-1}}|y| \exp \left\{-\frac{c_{2}|y|^{2} t}{u(t-u)}\right\} d y .
$$

Since the integral in the right-hand side of (3.24) is bounded by $c_{3} t^{-k / 2}$ where $c_{2}$ and $c_{3}$ are positive constants, we deduce as $t \rightarrow 0$ that

$$
\int_{\Omega} F_{2}(t, x, x) d x=\sum_{i=1}^{m} \int_{\partial \Omega_{\imath}} g(t, z) \gamma_{\imath}(z) d z+0\left(t^{(4-k) / 2}\right),
$$

where

$$
g(t, z)=\int_{0}^{t}(t-u) d u \int_{\partial \Omega_{2}} G_{N}(t-u, y, z) G_{N}(u, z, y) d y
$$


The right-hand side of (3.26) can be computed by taking the first term in the series expansion of the Neumann heat kernels (see [1])

$$
G_{N}(t-u, y, z)=2 q(t-u, y, z) \text { and } G_{N}(u, z, y)=2 q(u, z, y)
$$

where

$$
q(t, y, z)=(4 \pi t)^{-k / 2} \exp \left\{-\frac{|y-z|^{2}}{4 t}\right\}
$$

The explicit computation can be carried out with the help of a suitably chosen local coordinate system and the localization principle (see [1]). We leave the details of this computation to the interested reader and we content ourselves with the statement that the leading term of $g(t, z)$ is equal to the same integral in the Euclidean space, i.e,

$$
(4 \pi t)^{k / 2} g(t, z)=4 \int_{0}^{t}\left[\frac{t}{4 \pi u(t-u)}\right]^{k / 2}(t-u) d u \int_{R^{k-1}} \exp \left\{-\frac{|z-y|^{2}}{4(t-u)}-\frac{|y-z|^{2}}{4 u}\right\} d y+0\left(t^{2}\right) .
$$

After some reduction, we deduce that

$$
\begin{aligned}
(4 \pi t)^{k / 2} g(t, z) & =2\left(\frac{t}{\pi}\right)^{1 / 2} \int_{0}^{t}\left(\frac{t-u}{u}\right)^{1 / 2} d u+0\left(t^{2}\right) \\
& =\sqrt{\pi} t^{3 / 2}+0\left(t^{2}\right) .
\end{aligned}
$$

On inserting (3.29) into (3.25) we arrive at the proof of Lemma 2.

Finally, our result (2.1) follows immediately from (3.17), (3.18), (3 21) and the expansion of $\Theta_{N}(t)$ for the Neumann conditions on $\partial \Omega_{\imath}(i=1, \ldots, m)$.

\section{REFERENCES}

[1] HSU, P., On the $\theta$-function of a compact Riemannian manifold with boundary, C.R. Acad. Scl., Paris, Ser. I, 309 (1989), 507-510.

[2] KAC, M., Can one hear the shape of a drum? Amer. Math. Monthly, 73, Part II (1966), 1-23.

[3] McKEAN, H.P., Jr. and SINGER, I.M., Curvature and the eigenvalues of the Laplacian, J. Diff. Geom., 1 (1967), 43-69

[4] ZAYED, E.M.E. and YOUNIS, A.I., An inverse problem for a general convex domain with impedance boundary conditions, Quart. Applied Math. 48 (1) (1990), 181-188

[5] ZAYED, E.M.E., Hearing the shape of a general convex domain, J. Math. Anal. Appl., 142 (1989), 170-187

[6] ZAYED, E.M.E., Hearing the shape of a general convex domain An extension to higher dimensions, Portugaliae Math., 48 (1991), 259-280

[7] ZAYED, E.M.E., Heat equation for a general convex domain in $\mathrm{R}^{3}$ with a finite number of piecewise impedance boundary conditions, J. Applicable Anal., 42 (1991), 209-220. 


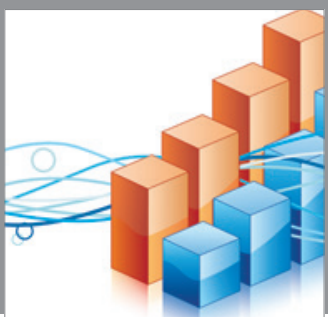

Advances in

Operations Research

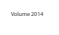

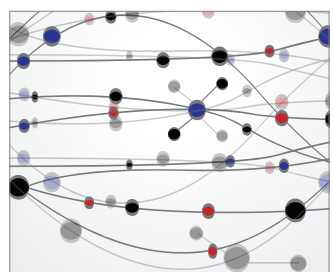

\section{The Scientific} World Journal
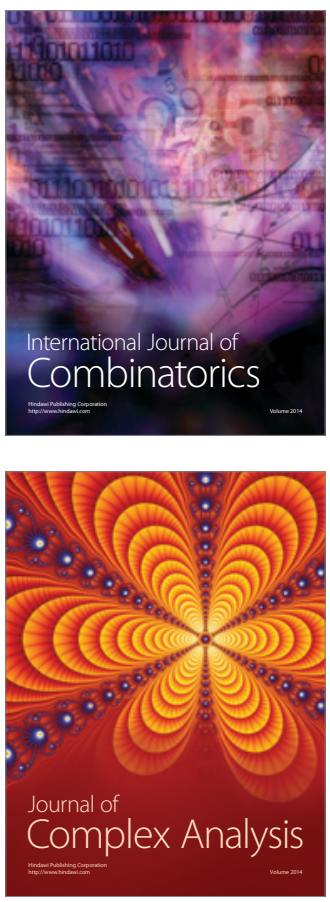

International Journal of

Mathematics and

Mathematical

Sciences
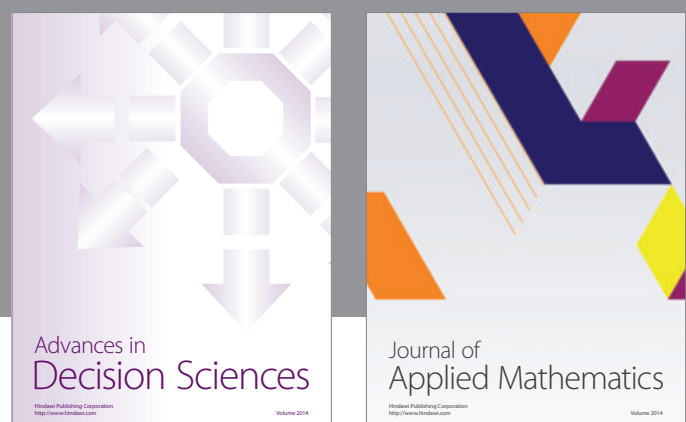

Journal of

Applied Mathematics
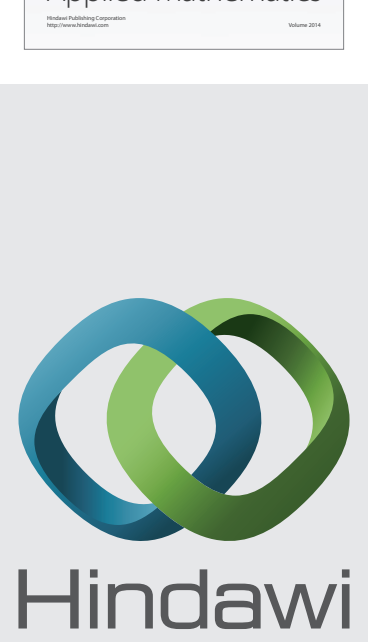

Submit your manuscripts at http://www.hindawi.com
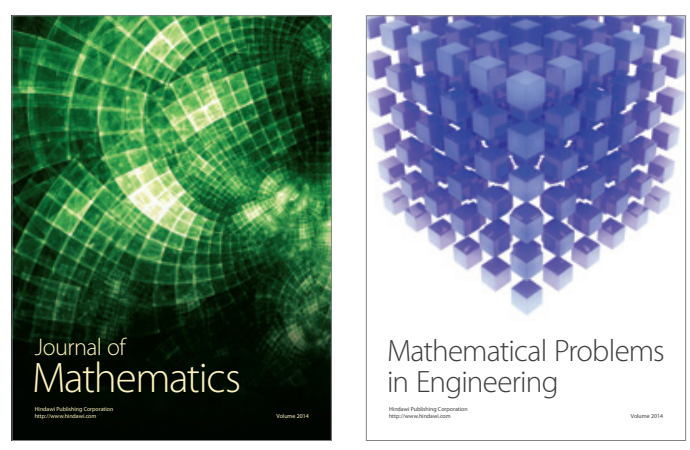

Mathematical Problems in Engineering
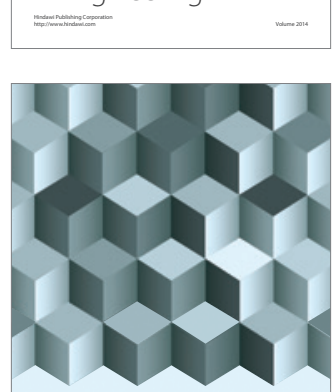

Journal of

Function Spaces
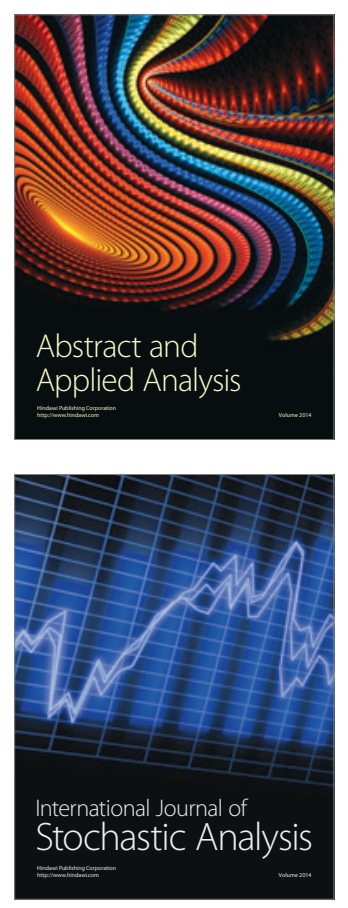

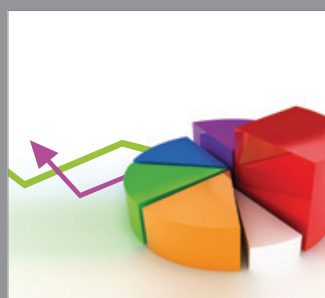

ournal of

Probability and Statistics

Promensencen
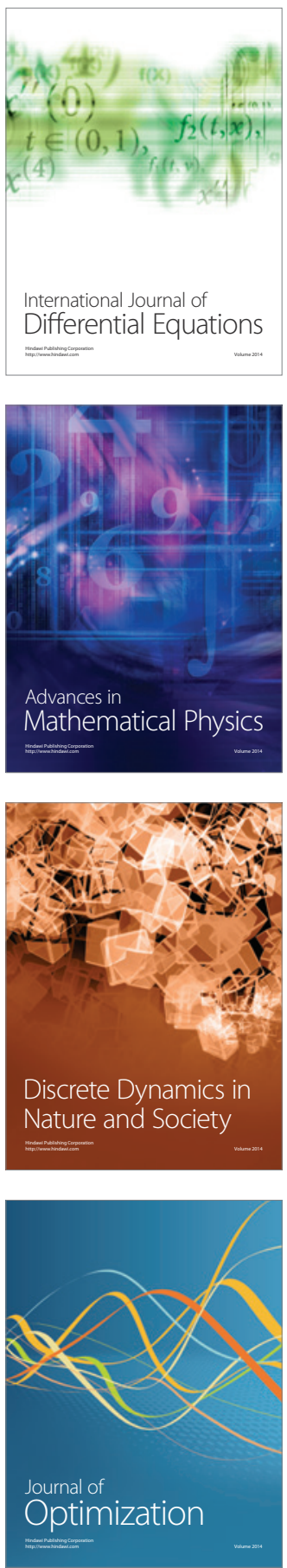